Göteborg ITP 97-11

June 1997

hep-th/9706044

\title{
Generalized BRST quantization and massive vector fields
}

\author{
Robert Marnelius: and Ikuo S. Sogami[2 \\ Institute of Theoretical Physics \\ Chalmers University of Technology \\ Göteborg University \\ S-412 96 Göteborg, Sweden
}

\begin{abstract}
A previously proposed generalized BRST quantization on inner product spaces for second class constraints is further developed through applications. This BRST method involves a conserved generalized BRST charge $Q$ which is not nilpotent $Q^{2} \neq 0$ but which satisfies $Q=\delta+\delta^{\dagger}, \delta^{2}=0$, and by means of which physical states are obtained from the projection $\delta|p h\rangle=\delta^{\dagger}|p h\rangle=0$. A simple model is analyzed in detail from which some basic properties and necessary ingredients are extracted. The method is then applied to a massive vector field. An effective theory is derived which is close to the one of the Stückelberg model. However, since the scalar field here is introduced in order to have inner product solutions, a massive Yang-Mills theory with polynomial interaction terms might be possible to construct.
\end{abstract}

\footnotetext{
${ }^{1}$ E-mail: tferm@fy.chalmers.se

${ }^{2}$ Address: Department of Physics, Kyoto Sangyo University, Kyoto 603, Japan

E-mail: sogami@cc.kyoto-su.ac.jp
} 


\section{Introduction.}

BRST quantization in the BFV formulation [1] is a consistent procedure to quantize general gauge theories i.e. systems with first class constraints in Dirac's constraint classification. Now there are many interesting models which naturally are formulated as systems with second class constraints. Massive vector fields and massive Yang-Mills are here important examples. In this paper we develop a generalized BRST quantization for systems of the latter type and at the end we treat the massive vector field as an example. The method raises some hopes that massive Yang-Mills may be quantized in a consistent fashion without a physical scalar boson.

Quantization of theories with second class constraints are usually avoided although such theories are possible to quantize. There are in fact three main roads to their quantization: 1) One may eliminate the constraints after which one obtains a regular quantum theory which, however, usually is no longer manifestly Lorentz invariant. A consistent renormalization procedure for such field theories seems therefore extremely difficult to set up. 2) One may formulate a modified theory which in a particular sense is equivalent to the original theory by turning the second class constraints into first class ones by means of auxiliary fields. ( A general method is given in [2].) This modified theory may then be quantized as an ordinary gauge theory. Notice, however, that the modified theory can never be exactly equivalent to the original theory. It may e.g. have different global symmetries. Massive Yang-Mills may be turned into a gauge theory by means of a so called Stückelberg transformation [3], which introduces auxiliary scalar fields. However, the resulting Lagrangian is nonlinear in these Stückelberg fields [4] and is therefore nonrenormalizable [5]. 3) The third possibility is to quantize the second class theory as a projection from an original state space spanned by the original variables: i) One may use the path integral formulation of Senjanovic [6] in which simply the measure $\sqrt{\operatorname{det}\left\{\phi_{a}, \phi_{b}\right\}} \prod_{a} \delta\left(\phi_{a}\right)$ is inserted where $\phi_{a}=0$ are the second class constraints. There are also more elaborate BRST approaches which are designed to avoid singular measures: ii) One approach involves two anticommuting nilpotent charges, $Q^{a}, a=1,2$, which are used to project out physical states according to $Q^{a}|p h\rangle=0[7$, 8]. The number of ghost fields in this approach is half the number of second class constraints. This method is applied to massive YangMills in [7]. iii) Another approach was proposed in [9, 10] and will be investigated in this paper.

In [9] one of us proposed the following generalized BRST formulation: If the constraints satisfy an algebra of the general form

$$
\left[\phi_{a}, \phi_{b}\right]=i U_{a b}^{c} \phi_{c}+i f_{a b},
$$

where $f_{a b}$ does not vanish on the constraint surface $\phi_{a}=0$, then we have a theory with second class constraints. A particular example is a gauge theory with anomalies in which case $f_{a b}$ is the anomaly $\left(f_{a b} \propto \hbar\right)$. The BRST charge is then constructed as if $f_{a b}$ is zero using the BFV algorithm [1]. The number of ghost fields is then always equal to the number of constraints. As a consequence the resulting BRST charge is not nilpotent. We have

$$
Q^{2}=\frac{1}{2} i f_{a b} C^{a} C^{b}+\cdots,
$$

where $C^{a}$ are the ghost fields. If $Q$ is conserved one may perform the projection

$$
Q|p h\rangle=0 .
$$


However, this is not a sufficient condition for a consistent theory as was shown in 11] where massive Yang-Mills was considered. In 10] an additional condition was proposed. This condition was introduced in order to have solutions which belong to inner product spaces. The additional requirements were that the BRST charge must be possible to decompose as follows

$$
Q=\delta+\delta^{\dagger}, \quad \delta^{2}=0
$$

and that (1.3) should be replaced by

$$
\delta|p h\rangle=\delta^{\dagger}|p h\rangle=0,
$$

where $\delta$ and $\delta^{\dagger}$ are independent and contain effectively half the constraints in $Q$ each. In the ordinary case when $Q$ is nilpotent (1.3) and (1.5) are equivalent. However, when $Q^{2} \neq 0(1.5)$ is more restrictive than (1.3), in other words (1.5) implies (1.3) but not vice versa. By means of a simple example it was demonstrated that the problem with (1.3) as pointed out in [1] is avoided by (1.5).

The above generalized BRST procedure for second class constraints is a straightforward generalization of the quantization procedure for general gauge theories as formulated in [10], 12]-17]. With the conditions (1.4) and (1.5) it has so far not been applied properly to any model. It has been applied to a trivial case in [10, and if one neglects the zero modes it was successfully applied to the free anomalous string in subcritical dimensions in [9, 18]. It has also partly been applied to the massive superparticle in [19]. Now the above generalized BRST quantization is very poorly developed. We have e.g. no simple algorithm which determines effective theories with generalized BRST invariance. In order to develop appropriate techniques we perform a detailed study of a simple model with finite number of degrees of freedom in section 2. This model contains some typical features which make the results generalizable to more involved models. In section 3 the results are applied to the massive vector field. We find then a simple effective theory which is similar to the Stückelberg model. It is described in section 4. Finally in section 5 we summarize our results and comment on massive Yang-Mills. Sections 2 and 3 are rather technical and awkward partly since even the corresponding treatment of the conventional case as given in [12] [17] is not fully developed. One may skip these sections in a first reading.

\section{A simple example}

Consider a nonrelativistic particle in two dimensions confined to move along a straight line. A simple Lagrangian for this system is

$$
L=\frac{1}{2}\left(\dot{x}^{2}+\dot{y}^{2}\right)-\lambda\left(x \sin \theta_{0}-y \cos \theta_{0}\right),
$$

where $\lambda$ is a Lagrange multiplier and $\theta_{0}$ a fixed angle. Variation with respect to $\lambda$ yields the constraint $\phi_{1}=0$, where

$$
\phi_{1} \equiv x \sin \theta_{0}-y \cos \theta_{0},
$$


which means that the particle is confined to move along a line through the origin with angle $\theta_{0}$ relative to the $x$-axis. The corresponding Hamiltonian is

$$
H=\dot{x} p_{x}+\dot{y} p_{y}-L=\frac{1}{2}\left(p_{x}^{2}+p_{y}^{2}\right)+\lambda\left(x \sin \theta_{0}-y \cos \theta_{0}\right)
$$

and in addition we have the primary constraint

$$
P_{\lambda} \equiv \frac{\partial L}{\partial \lambda}=0
$$

The total Hamiltonian is therefore $H_{T}=H+v P_{\lambda}$. The consistency condition $\dot{P}_{\lambda}=$ $\left\{P_{\lambda}, H_{T}\right\}=0$ etc yield then $v=0$ and the complete set of constraint variables; $P_{\lambda}, \lambda, \phi_{1}$, $\phi_{2}$, where $\phi_{1}$ is given by (2.2) and

$$
\phi_{2} \equiv p_{x} \sin \theta_{0}-p_{y} \cos \theta_{0}
$$

All these constraints are of second class.

We turn now to the quantum theory of the above system. We impose therefore the canonical commutation relations (the nonzero part)

$$
\left[x, p_{x}\right]_{-}=\left[y, p_{y}\right]_{-}=\left[\lambda, P_{\lambda}\right]_{-}=i
$$

which yields

$$
\left[\phi_{1}, \phi_{2}\right]_{-}=i
$$

for the operators $\phi_{1,2}$. The algebra of the constraint operators $P_{\lambda}, \lambda, \phi_{1}, \phi_{2}$ is, thus, of the form (1.1) with $U_{a b}^{c}=0$. The form of the generalized BRST operator is, therefore, that of an abelian gauge theory according to the rules of [9]. This means that we have to insert a ghost for every constraint. We may write $(\mathrm{a}=1,2)$

$$
Q=\mathcal{C}^{a} \phi_{a}+\overline{\mathcal{P}}^{1} \lambda+\overline{\mathcal{P}}^{2} P_{\lambda}
$$

where the fermionic ghost operators $\mathcal{C}^{a}, \mathcal{P}_{b}, \overline{\mathcal{C}}_{b}, \overline{\mathcal{P}}^{a}$ satisfy the anticommutation relations (the nonzero part)

$$
\left[\mathcal{C}^{a}, \mathcal{P}_{b}\right]_{+}=\left[\overline{\mathcal{C}_{b}}, \overline{\mathcal{P}}^{a}\right]_{+}=\delta_{b}^{a}
$$

This BRST operator has the property

$$
Q^{2}=i\left(\mathcal{C}^{1} \mathcal{C}^{2}+\overline{\mathcal{P}}^{1} \overline{\mathcal{P}}^{2}\right)
$$

In (2.8) we have split the ghosts into two equally large sets, one of which we denote as ghosts and one of which we consider as conjugate momenta to antighosts $\left(\overline{\mathcal{C}}_{a}\right)$. The reason for this split is due to the condition (1.4). In the ordinary case it was found that (1.4) may always be satisfied if one considers dynamical Lagrange multipliers and antighosts which are equal in number to the basic (usually nontrivial) constraints and ghosts. (We have then e.g. $Q=\mathcal{C} \phi_{1}+P_{\lambda} \overline{\mathcal{P}}$ which describes a first class system.) Notice, however, that the split in ghosts and conjugate momenta to antighosts in (2.8) only is tentative. In fact, later we will find that it is not the most natural one. 
In the present case we expect (1.4) to be satisfied if the second class constraints may be split into equally large sets one of which fixes the Lagrange multipliers. In fact, for our model (2.1) the condition (1.4) is satisfied by expressions of the form

$$
\delta=g^{a} \psi_{a}^{\dagger}
$$

where $\psi_{a}, a=1,2$, are two complex bosonic constraint operators and $g^{a}, a=1,2$, two complex fermionic ghost operators which may be chosen to satisfy

$$
\begin{aligned}
& {\left[g^{a}, g^{b}\right]=\left[g^{a}, g^{\dagger b}\right]=0,} \\
& {\left[\psi_{1}, \psi_{2}\right]=\left[\psi_{1}, \psi_{1}^{\dagger}\right]=\left[\psi_{2}, \psi_{2}^{\dagger}\right]=0, \quad\left[\psi_{1}, \psi_{2}^{\dagger}\right]=2 i}
\end{aligned}
$$

Explicit realizations are e.g.

$$
g^{a}=\frac{1}{2}\left(\mathcal{C}^{a}-i \overline{\mathcal{P}}^{a}\right), \quad \psi_{1}=\phi_{1}-i \lambda, \quad \psi_{2}=\phi_{2}-i P_{\lambda}
$$

or

$$
g^{1}=\frac{1}{2}\left(\mathcal{C}^{1}+i \overline{\mathcal{P}}^{2}\right), \quad g^{2}=\frac{1}{2}\left(\mathcal{C}^{2}-i \overline{\mathcal{P}}^{1}\right), \quad \psi_{1}=\phi_{1}+i P_{\lambda}, \quad \psi_{2}=\phi_{2}-i \lambda .
$$

In fact, there are infinitely many ways in which (2.11) and (2.12) may be realized. To see this one may observe that $Q$ is invariant under the scale transformations $\left(\gamma_{1}\right.$ and $\gamma_{2}$ are real parameters and $U_{1,2}$ unitary operators)

$$
\begin{aligned}
U_{1}\left(\gamma_{1}\right): & \phi_{1} \rightarrow e^{\gamma_{1}} \phi_{1}, \quad \phi_{2} \rightarrow e^{-\gamma_{1}} \phi_{2}, \quad \mathcal{C}^{1} \rightarrow e^{-\gamma_{1}} \mathcal{C}^{1}, \quad \mathcal{C}^{2} \rightarrow e^{\gamma_{1}} \mathcal{C}^{2}, \\
& \mathcal{P}_{1} \rightarrow e^{\gamma_{1}} \mathcal{P}_{1}, \quad \mathcal{P}_{2} \rightarrow e^{-\gamma_{1}} \mathcal{P}_{2}, \\
U_{2}\left(\gamma_{2}\right): & \lambda \rightarrow e^{\gamma_{2}} \lambda, \quad P_{\lambda} \rightarrow e^{-\gamma_{2}} P_{\lambda}, \quad \overline{\mathcal{P}}^{1} \rightarrow e^{-\gamma_{2}} \overline{\mathcal{P}}^{1}, \quad \overline{\mathcal{P}}^{2} \rightarrow e^{\gamma_{2}} \overline{\mathcal{P}}^{2}, \\
& \overline{\mathcal{C}}_{1} \rightarrow e^{\gamma_{2}} \overline{\mathcal{C}}_{1}, \quad \overline{\mathcal{C}}_{2} \rightarrow e^{-\gamma_{2}} \overline{\mathcal{C}}_{2},
\end{aligned}
$$

and under the rotations ( $\theta_{1}$ and $\theta_{2}$ are real parameters and $R_{1,2}$ unitary operators)

$$
\begin{aligned}
R_{1}\left(\theta_{1}\right): \quad & \phi_{1} \rightarrow \cos \theta_{1} \phi_{1}+\sin \theta_{1} \phi_{2}, \quad \phi_{2} \rightarrow \cos \theta_{1} \phi_{2}-\sin \theta_{1} \phi_{1}, \\
& \mathcal{C}^{1} \rightarrow \cos \theta_{1} \mathcal{C}^{1}+\sin \theta_{1} \mathcal{C}^{2}, \quad \mathcal{C}^{2} \rightarrow \cos \theta_{1} \mathcal{C}^{2}-\sin \theta_{1} \mathcal{C}^{1}, \\
& \mathcal{P}_{1} \rightarrow \cos \theta_{1} \mathcal{P}_{1}+\sin \theta_{1} \mathcal{P}_{2}, \quad \mathcal{P}_{2} \rightarrow \cos \theta_{1} \mathcal{P}_{2}-\sin \theta_{1} \mathcal{P}_{1}, \\
R_{2}\left(\theta_{2}\right): \quad & \lambda \rightarrow \cos \theta_{2} \lambda+\sin \theta_{2} P_{\lambda}, \quad P_{\lambda} \rightarrow \cos \theta_{2} P_{\lambda}-\sin \theta_{2} \lambda \\
& \overline{\mathcal{P}}^{1} \rightarrow \cos \theta_{2} \overline{\mathcal{P}}^{1}+\sin \theta_{2} \overline{\mathcal{P}}^{2}, \quad \overline{\mathcal{P}}^{2} \rightarrow \cos \theta_{2} \overline{\mathcal{P}}^{2}-\sin \theta_{2} \overline{\mathcal{P}}^{1}, \\
& \overline{\mathcal{C}}_{1} \rightarrow \cos \theta_{2} \overline{\mathcal{C}}_{1}+\sin \theta_{2} \overline{\mathcal{C}}_{2}, \quad \overline{\mathcal{C}}_{2} \rightarrow \cos \theta_{2} \overline{\mathcal{C}}_{2}-\sin \theta_{2} \overline{\mathcal{C}}_{1} .
\end{aligned}
$$

These transformations allow us, therefore, to represent $g^{a}$ and $\psi_{a}$ in (2.11) in terms of four parameters starting from either (2.13) or (2.14). (Notice that (2.13) and (2.14) are related by an $R_{2}$-rotation in 90 degrees.) However, this does not exhaust all possibilities. We may also consider representations (2.11) in (1.4) where

$$
\begin{aligned}
& g^{1}=\frac{1}{2}\left(\mathcal{C}^{1}+i \mathcal{C}^{2}\right), \quad g^{2}=\frac{1}{2}\left(\overline{\mathcal{P}}^{1}+i \overline{\mathcal{P}}^{2}\right), \\
& \psi_{1}=\phi_{1}+i \phi_{2}, \quad \psi_{2}=\lambda+i P_{\lambda} .
\end{aligned}
$$

Instead of 2.12) $\psi_{a}$ satisfy here $\left[\psi_{a}, \psi_{b}^{\dagger}\right]=2 \delta_{a b}$. Again the transformations (2.15) and (2.16) allow us to represent $g^{a}$ and $\psi_{a}$ in (2.11) in terms of four parameters but now starting from (2.17) which is different from the previous representation. 
Using (1.4) with the representation (2.11) the solutions with nonzero norms of the conditions (1.5) may be shown to satisfy

$$
\text { i) } g^{a}|p h\rangle=0, \quad \psi_{a}|p h\rangle=0
$$

or

$$
\text { ii) } g^{a \dagger}|p h\rangle=0, \quad \psi_{a}^{\dagger}|p h\rangle=0
$$

Both these sets of solutions should be physically equivalent. (Notice that the first conditions in (2.18) and (2.19) by themselves imply $Q^{2}|\mathrm{ph}\rangle=0$.)

We shall now show that the nontrivial solutions of (1.5), i.e. the solutions up to zero norm states, may be represented in the form

$$
|s\rangle=e^{\Lambda}|\phi\rangle
$$

where $\Lambda$ is an Hermitian operator and $|\phi\rangle$ a simple state determined by Hermitian conditions. In the ordinary case we have the representation (2.20) where $|s\rangle$ is a BRST singlet, $\Lambda=[Q, \psi]$ where $\psi$ is a gauge fixing fermion, and where $|\phi\rangle$ is determined by a complete irreducible set of BRST doublets in involution [15]. Since the BRST charge is no longer nilpotent in this generalized BRST case, operators and states are decomposed into higher multiplets than doublets. For our simple model the basic variables may be decomposed into BRST triplets. In fact, we have the following elementary hermitian BRST triplets

$$
\begin{array}{llll}
(1) & \left\{\mathcal{P}_{1}, \phi_{1}, \mathcal{C}^{2}\right\}, & (2) & \left\{\mathcal{P}_{2}, \phi_{2}, \mathcal{C}^{1}\right\} \\
(3) & \left\{\overline{\mathcal{C}}_{2}, P_{\lambda}, \overline{\mathcal{P}}^{1}\right\}, & (4) & \left\{\overline{\mathcal{C}}_{1}, \lambda, \overline{\mathcal{P}}^{2}\right\}
\end{array}
$$

where the entries $(a, b, c)$ are related by $b \propto[Q, a], c \propto[Q, b]$. All these triplets form generalized quartets or rather sixtets. However, half of them are in involution. In fact the following four combinations of two of the above triplets are in involution:

$$
[1]:(1)+(3), \quad[2]:(2)+(4), \quad[3]:(1)+(4), \quad[4]:(2)+(3) .
$$

The most simple BRST invariant solutions of the form (2.20) should therefore be

$$
|s\rangle_{l}=e^{\Lambda_{l}}|\phi\rangle_{l}, \quad l=1,2,3,4,
$$

where $|\phi\rangle_{l}$ is a state determined by the operators in the combination $[l]$ in $(2.22)$ i.e.

$$
\begin{aligned}
& \mathcal{P}_{1}|\phi\rangle_{1}=\phi_{1}|\phi\rangle_{1}=\mathcal{C}^{2}|\phi\rangle_{1}=\overline{\mathcal{C}}_{2}|\phi\rangle_{1}=P_{\lambda}|\phi\rangle_{1}=\overline{\mathcal{P}}^{1}|\phi\rangle_{1}=0, \\
& \mathcal{P}_{2}|\phi\rangle_{2}=\phi_{2}|\phi\rangle_{2}=\mathcal{C}^{1}|\phi\rangle_{2}=\overline{\mathcal{C}}_{1}|\phi\rangle_{2}=\lambda|\phi\rangle_{2}=\overline{\mathcal{P}}^{2}|\phi\rangle_{2}=0, \\
& \mathcal{P}_{1}|\phi\rangle_{3}=\phi_{1}|\phi\rangle_{3}=\mathcal{C}^{2}|\phi\rangle_{3}=\overline{\mathcal{C}}_{1}|\phi\rangle_{3}=\lambda|\phi\rangle_{3}=\overline{\mathcal{P}}^{2}|\phi\rangle_{3}=0, \\
& \mathcal{P}_{2}|\phi\rangle_{4}=\phi_{2}|\phi\rangle_{4}=\mathcal{C}^{1}|\phi\rangle_{4}=\overline{\mathcal{C}}_{2}|\phi\rangle_{4}=P_{\lambda}|\phi\rangle_{4}=\overline{\mathcal{P}}^{1}|\phi\rangle_{4}=0 .
\end{aligned}
$$

If we choose

$$
\begin{aligned}
& \Lambda_{1}=\phi_{2} \lambda-i \overline{\mathcal{P}}^{2} \mathcal{P}_{2}+i \mathcal{C}^{1} \overline{\mathcal{C}}_{1} \\
& \Lambda_{2}=-\phi_{1} P_{\lambda}-i \overline{\mathcal{P}}^{1} \mathcal{P}_{1}+i \mathcal{C}^{2} \overline{\mathcal{C}}_{2} \\
& \Lambda_{3}=\phi_{2} P_{\lambda}+i \overline{\mathcal{P}}^{1} \mathcal{P}_{2}+i \mathcal{C}^{1} \overline{\mathcal{C}}_{2} \\
& \Lambda_{4}=\phi_{1} \lambda-i \mathcal{C}^{2} \overline{\mathcal{C}}_{1}-i \overline{\mathcal{P}}^{2} \mathcal{P}_{1}
\end{aligned}
$$


in (2.23) then $|s\rangle_{1,2}$ satisfy $(2.18)$ in the realization (2.13) and $|s\rangle_{3,4}$ satisfy (2.18) in the realization (2.14). Notice that $\left[Q, \Lambda_{l}\right]=0, Q|\phi\rangle_{l}=0$, which implies $Q|s\rangle_{l}=0$. Notice also that the conditions (2.24) are invariant under the scale transformations (2.15) but not under the rotations (2.16) except for rotations in 180 degrees. Furthermore, they are related by the rotations (2.16). Starting from e.g. $|s\rangle_{1}=e^{\Lambda_{1}}|\phi\rangle_{1}$ one may by successive rotations in 90 degrees reach all the four cases above. Arbitrary rotations yield singlets which interpolate the ones in (2.23). The unitary scale transformations (2.15) scale $\Lambda_{l}$ according to

$$
\Lambda_{l} \rightarrow \alpha_{l} \Lambda_{l}, \quad \alpha_{1}=e^{-\gamma_{1}+\gamma_{2}}, \quad \alpha_{2}=e^{\gamma_{1}-\gamma_{2}}, \quad \alpha_{3}=e^{-\gamma_{1}-\gamma_{2}}, \quad \alpha_{4}=e^{\gamma_{1}+\gamma_{2}} .
$$

Now, in distinction to the ordinary BRST case these unitary scale transformations, $U_{1}$ and $U_{2}$, do not yield unity on the $|\phi\rangle_{l}$-states. Instead we have

$$
|\phi\rangle_{l} \rightarrow \frac{1}{\sqrt{\alpha_{l}}}|\phi\rangle_{l} .
$$

Unitary equivalent solutions to (2.23) may therefore be written in the following form

$$
|s\rangle_{l}=\frac{1}{\sqrt{\alpha_{l}}} e^{ \pm \alpha_{l} \Lambda_{l}}|\phi\rangle_{l}, \quad l=1,2,3,4
$$

where $|\phi\rangle_{l}$ is the same solution of (2.24) as in (2.23). (Notice, however, that $|\phi\rangle_{l}$ scaled with any constant still satisfy (2.24).) Here as well as in the ordinary BRST case $|\phi\rangle_{l}$ is not an inner product space. However, while $|\phi\rangle_{l}$ in the ordinary case may be regularized to an inner product state it is here regularized to a zero norm state which is the reason why the property (2.27) is allowed for unitary transformations here. The reason behind this difference compared to the ordinary case is that our scheme in a sense makes use of an unnecessary large number of ghost variables for second class constraints. (A scheme with a nilpotent charge like the one in [7, 8] should not have the property (2.27).) Obviously, the measure operator $e^{\Lambda_{l}}$ acts here in a more nontrivial way as compared to the ordinary case since a scale transformation of $\Lambda_{l}$ affects the norms. (In the ordinary case $e^{\Lambda_{l}}$ is just a regulator.)

The above properties may be elaborated further. The $\Lambda_{l}$ operators in (2.25) satisfy the properties

$$
\Lambda_{l}|\phi\rangle_{l^{\prime}}=0 \text { for } l \neq l^{\prime}
$$

Furthermore, $\Lambda_{1}$ and $\Lambda_{2}$ as well as $\Lambda_{3}$ and $\Lambda_{4}$ satisfy a closed algebra. Consider the following two identifications

$$
\begin{aligned}
& \text { (1) } K_{1}=\Lambda_{1}, \quad K_{2}=\Lambda_{2}, \quad K_{3}=\left[\Lambda_{1}, \Lambda_{2}\right], \\
& \text { (2) } K_{1}=\Lambda_{3}, \quad K_{2}=\Lambda_{4}, \quad K_{3}=\left[\Lambda_{3}, \Lambda_{4}\right] .
\end{aligned}
$$

For both cases, (1) and (2), we have then

$$
K_{3}=\left[K_{1}, K_{2}\right], \quad\left[K_{3}, K_{1}\right]=2 K_{1}, \quad\left[K_{3}, K_{2}\right]=-2 K_{2},
$$

which is an $\mathrm{SL}(2, \mathrm{R})$ algebra. The $K_{3}$ operator in the two cases satisfies the properties

$$
\text { (1) } K_{3}|\phi\rangle_{1}=-|\phi\rangle_{1}, \quad K_{3}|\phi\rangle_{2}=|\phi\rangle_{2}, \quad K_{3}|\phi\rangle_{3,4}=0
$$




$$
\text { (2) } \quad K_{3}|\phi\rangle_{1,2}=0, \quad K_{3}|\phi\rangle_{3}=-|\phi\rangle_{3}, \quad K_{3}|\phi\rangle_{4}=|\phi\rangle_{4}
$$

By means of these properties we may construct the following more general expressions of the solutions (2.23) (cf. the corresponding constructions for general gauge theories in [16]):

$$
|s\rangle_{1,2}=e^{\alpha \Lambda_{1}+\beta \Lambda_{2}}|\phi\rangle_{1,2}, \quad|s\rangle_{3,4}=e^{\alpha \Lambda_{3}+\beta \Lambda_{4}}|\phi\rangle_{3,4} .
$$

These expressions may be reduced to the form (2.28) by means of the following factorizations

$$
e^{\alpha K_{1}+\beta K_{2}}=e^{\delta_{1} K_{1}} e^{\delta_{2} K_{2}} e^{\delta_{3} K_{3}}=e^{\delta_{2}^{\prime} K_{2}} e^{\delta_{1}^{\prime} K_{1}} e^{\delta_{3}^{\prime} K_{3}}
$$

where

$$
e^{-\delta_{3}}=\cosh \sqrt{\alpha \beta}, \quad \delta_{1}=\alpha \frac{\tanh \sqrt{\alpha \beta}}{\sqrt{\alpha \beta}}, \quad \delta_{2}=\frac{1}{2} \beta \frac{\sinh 2 \sqrt{\alpha \beta}}{\sqrt{\alpha \beta}}
$$

and

$$
e^{\delta_{3}^{\prime}}=\cosh \sqrt{\alpha \beta}, \quad \delta_{1}^{\prime}=\frac{1}{2} \alpha \frac{\sinh 2 \sqrt{\alpha \beta}}{\sqrt{\alpha \beta}}, \quad \delta_{2}^{\prime}=\beta \frac{\tanh \sqrt{\alpha \beta}}{\sqrt{\alpha \beta}}
$$

for $\alpha \beta>0$. For $\alpha \beta<0$ we have the same expressions with the corresponding trigonometric functions and $\sqrt{\alpha \beta}$ replaced by $\sqrt{-\alpha \beta}$. Eq. 2.36) inserted into (2.35) yields by means of (2.29), (2.33) and (2.34):

$$
\begin{aligned}
& |s\rangle_{1}=\lambda e^{\delta \alpha \Lambda_{1}}|\phi\rangle_{1}, \quad|s\rangle_{2}=\lambda e^{\delta \beta \Lambda_{2}}|\phi\rangle_{2} \\
& |s\rangle_{3}=\lambda e^{\delta \alpha \Lambda_{3}}|\phi\rangle_{3}, \quad|s\rangle_{4}=\lambda e^{\delta \beta \Lambda_{4}}|\phi\rangle_{4},
\end{aligned}
$$

where

$$
\lambda=\cosh \sqrt{\alpha \beta}, \quad \delta=\frac{\tanh \sqrt{\alpha \beta}}{\sqrt{\alpha \beta}} .
$$

Thus, in order for the expressions (2.35) to have the same normalizations as (2.28) when using the same $|\phi\rangle_{l}$-states we have to multiply $|s\rangle_{1,3}$ and $|s\rangle_{2,4}$ in (2.35) by the factor $\sqrt{2 \sqrt{\beta / \alpha} / \sinh 2 \sqrt{\alpha \beta}}$ and $\sqrt{2 \sqrt{\alpha / \beta} / \sinh 2 \sqrt{\alpha \beta}}$ respectively. These results may be directly compared with the corresponding results for general gauge theories [16]. The main difference is that for general gauge theories we have $K_{3}|\phi\rangle_{l}=0$ and $\lambda=1$. (Notice that $K_{i}$ in [16] are different from ours. To compare one has here to make the replacement $K_{1} \rightarrow-K_{2}, K_{2} \rightarrow K_{1}$ implying $\alpha \rightarrow-\beta, \beta \rightarrow \alpha$.)

In order to determine the time evolution of the BRST invariant solutions we have to determine an appropriate BRST invariant Hamiltonian. The original Hamiltonian operator is

$$
H=\frac{1}{2}\left(p_{x}^{2}+p_{y}^{2}\right)+\lambda \phi_{1}
$$

which is not BRST invariant since

$$
[Q, H]=i\left(\mathcal{C}^{1} \phi_{2}-\mathcal{C}^{2} \lambda-\phi_{1} \overline{\mathcal{P}}^{2}\right)
$$


A BRST invariant Hamiltonian may, however, be obtained by adding ghosts to $H$. We have then

$$
H_{\mathrm{BRST}}=H+i \mathcal{P}_{1} \overline{\mathcal{P}}^{2}+i \overline{\mathcal{C}}_{1} \mathcal{C}^{2}-i \mathcal{P}_{2} \mathcal{C}^{1}
$$

which is one possible candidate of a BRST invariant Hamiltonian. Other candidates are obtained by truncation. We have

$$
H_{\mathrm{BRST}}^{\prime}=\frac{1}{2}\left(p_{x}^{2}+p_{y}^{2}\right)-i \mathcal{P}_{2} \mathcal{C}^{1}
$$

by first removing the $\lambda \phi_{1}$-term in the original Hamiltonian 2.41). Furthermore, since

$$
\frac{1}{2}\left(p_{x}^{2}+p_{y}^{2}\right)=\frac{1}{2}\left(p_{x} \cos \theta_{0}+p_{y} \sin \theta_{0}\right)^{2}+\frac{1}{2}\left(\phi_{2}\right)^{2}
$$

we have also the following completely ghost independent BRST invariant Hamiltonian

$$
H_{\mathrm{BRST}}^{\prime \prime}=\frac{1}{2}\left(p_{x} \cos \theta_{0}+p_{y} \sin \theta_{0}\right)^{2} .
$$

A time dependent BRST invariant solution is naturally expected to be given by

$$
|s, t\rangle=e^{-i H_{0} t}|s\rangle
$$

where $H_{0}$ is one of the the BRST invariant Hamiltonians (2.43), (2.44) or (2.46). Which one should be chosen? From the results obtained for ordinary BRST quantization [13] the appropriate Hamiltonian should be such that we also have $|\phi, t\rangle=e^{-i H_{0} t}|\phi\rangle$. This requires $H_{0}$ to commute with $\Lambda_{l}$. We notice then that $H_{\text {BRST }}$ in (2.43) does not commute with any $\Lambda_{l}$. $H_{\mathrm{BRST}}^{\prime}$ in (2.44) commutes with $\Lambda_{1}$ and $\Lambda_{3}$ but not with $\Lambda_{2}$ and $\Lambda_{4}$, while only $H_{\mathrm{BRST}}^{\prime \prime}$ in (2.46) commutes with all $\Lambda_{l}$. Notice also that only $H_{\mathrm{BRST}}^{\prime \prime}$ is invariant under the scaling (2.15) and under the rotations (2.16). $H_{\mathrm{BRST}}^{\prime \prime}$ seems therefore to be the appropriate choice for $H_{0}$. (All this should be viewed from the corresponding reparametrization invariant theory whose BRST treatment allows for transformations between the Hamiltonian and the constraints [17.)

In order to be able to relate the above results to path integral formulations we have to determine appropriate quantization rules. From (2.12),(2.18) and (2.19) we conclude that the unphysical part of the original state space is spanned by oscillator variables satisfying the algebra (the nonzero elements)

$$
\left.\mid g^{a}, k_{b}^{\dagger}\right]_{+}=\delta_{b}^{a}, \quad\left[A, B^{\dagger}\right]_{-}=1
$$

where $g^{a}, k_{a}, a=1,2$, are fermionic and $A$ and $B$ bosonic. $g^{a}$ may be identified with $g^{a}$ in (2.11), and $A$ and $B$ with $\psi_{1}\left(\psi_{2}\right)$ and $\frac{1}{2} i \psi_{2}\left(-\frac{1}{2} i \psi_{1}\right)$ in (2.12). The corresponding Fock representation consists when diagonalized of half positive and half indefinite metric oscillators both for bosons and fermions separately. This implies in turn that half the hermitian unphysical operators have imaginary eigenvalues [14]. For the bosons, $x$ and $y$, we should have real eigenvalues since they are treated on equal level which implies that the Lagrange multiplier $\lambda$ should have imaginary eigenvalues. Since the BRST invariant Hamiltonian $H_{0}$ should have real eigenvalues $H_{0}=H_{B R S T}$ is excluded. For the fermions a natural choice is such that $\Lambda_{l}$ has imaginary eigenvalues. This implies that the ghost 
variables $\mathcal{C}^{a}$ and $\overline{\mathcal{C}}_{a}$ should have opposite properties, one should have real eigenvalues and the other imaginary ones.

Relation (2.47) and the form (2.35) for the solutions lead to the following transition amplitudes $\left(H_{0}\right.$ and $\Lambda_{l}$ commute)

$$
\left\langle s^{\prime}, t^{\prime} \mid s, t\right\rangle=\left\langle s^{\prime}\left|e^{i H_{0}\left(t^{\prime}-t\right)}\right| s\right\rangle=\left\{\begin{array}{cc}
{ }_{l}\left\langle\phi^{\prime}\left|e^{i H_{0}\left(t^{\prime}-t\right)+\alpha \Lambda_{1}+\beta \Lambda_{2}}\right| \phi\right\rangle_{l}, & l=1,2 \\
l\left\langle\phi^{\prime}\left|e^{i H_{0}\left(t^{\prime}-t\right)+\alpha \Lambda_{3}+\beta \Lambda_{4}}\right| \phi\right\rangle_{l}, & l=3,4
\end{array}\right.
$$

Replacing $\alpha$ and $\beta$ by $\alpha \rightarrow\left(t^{\prime}-t\right) \alpha$ and $\beta \rightarrow\left(t^{\prime}-t\right) \beta$ we are then led to the path integral representation (cf [13])

$$
\begin{aligned}
& \left\langle s^{\prime}, t^{\prime} \mid s, t\right\rangle=\int d q^{\prime} d q \phi^{\prime *}\left(q^{\prime *}\right) \phi(q)\left\langle q^{\prime}, t^{\prime} \mid q^{*}, t\right\rangle, \\
& \left\langle q^{\prime}, t^{\prime} \mid q^{*}, t\right\rangle=\int[d q][d p] e^{i \int_{t}^{t^{\prime}}\left(p \dot{q}-H_{\mathrm{eff}}\right) d t},
\end{aligned}
$$

where $q$ denotes collectively all the coordinates $\left(x, y, \lambda, \mathcal{C}^{a}, \overline{\mathcal{C}_{a}}\right)$ and $p$ their corresponding conjugate momenta. According to the previous results the normalization of these time dependent solutions is also time dependent. In order to have time independent normalization (2.49) has to be multiplied by the factor $1 / \sinh 2\left(t^{\prime}-t\right) \sqrt{\alpha \beta}$. Notice that (2.24) are conditions which act like boundary conditions in the path integral. $H_{\text {eff }}$ is an effective pseudoclassical Hamiltonian. For $H_{0}=H_{\mathrm{BRST}}^{\prime \prime}$ we have the following choices

$$
\begin{aligned}
& l=1,2: \quad H_{\mathrm{eff}}=\frac{1}{2}\left(p_{x} \cos \theta_{0}+p_{y} \sin \theta_{0}\right)^{2}+\alpha \lambda_{1}+\beta \lambda_{2}, \\
& l=3,4: \quad H_{\mathrm{eff}}=\frac{1}{2}\left(p_{x} \cos \theta_{0}+p_{y} \sin \theta_{0}\right)^{2}+\alpha \lambda_{3}+\beta \lambda_{4},
\end{aligned}
$$

where $\lambda_{l}$ are real pseudoclassical expressions from $\Lambda_{l}$. The choice of effective Hamiltonian depends on the choice of boundary condition. In fact, by means of the rotations (2.16) we may also obtain $H_{\text {eff }}$ and boundary conditions which interpolate between the above choices. One may convince oneself that for any choice of an allowed effective Hamiltonian in (2.50) together with the corresponding boundary condition implied by (2.24) and its interpolations by means of $(2.16)$ we get one and the same result: Integrations over the Lagrange multiplier and its conjugate momentum produce delta functions so that we for any choice effectively have

$$
\begin{aligned}
& \left\langle x^{\prime}, y^{\prime}, t^{\prime} \mid x, y, t\right\rangle= \\
& =\int[d x][d y]\left[d p_{x}\right]\left[d p_{y}\right] \delta\left(\phi_{1}\right) \delta\left(\phi_{2}\right) e^{i \int_{t}^{t^{\prime}}\left(p_{x} \dot{x}+p_{y} \dot{y}-\frac{1}{2}\left(p_{x} \cos \theta_{0}+p_{y} \sin \theta_{0}\right)^{2}\right)},
\end{aligned}
$$

which is in agreement with the prescription of [6].

Now the above effective Hamiltonians are not the natural ones from a geometrical point of view. They are also not "gauge fixed" in the sense that the corresponding Lagrangians are not regular. In order to find out what a more natural effective Hamiltonian requires we notice now the following relations between the Hamiltonians (2.43), (2.44) and (2.46).

$$
H_{B R S T}=H_{B R S T}^{\prime}+\Lambda_{4}=H_{B R S T}^{\prime \prime}+\frac{1}{2} i\left[\Lambda_{3}, \Lambda_{1}\right]+\Lambda_{4} .
$$


Thus, it seems that in order to have an effective Hamiltonian corresponding to (2.43) we must consider solutions of the following form

$$
|s\rangle=e^{\alpha\left(\Lambda_{4}+\frac{1}{2} i\left[\Lambda_{3}, \Lambda_{1}\right]\right)}|\phi\rangle_{4} .
$$

However, since $\frac{1}{2} i\left[\Lambda_{3}, \Lambda_{1}\right]$ has real eigenvalues and $\Lambda_{4}$ imaginary ones this leads to a complex effective Hamiltonian in the path integral. Furthermore, the corresponding effective Lagrangian is not regular.

Now the forms (2.23) and (2.35) of the solutions are not the only ones. By means of the representation (2.17) in (2.11) we have also

$$
|s\rangle_{l}=e^{B_{l}}|\phi\rangle_{l},
$$

where

$$
\begin{aligned}
& B_{1}=\alpha \frac{1}{2} i\left[\Lambda_{1}, \Lambda_{3}\right]-\beta \frac{1}{2} i\left[\Lambda_{1}, \Lambda_{4}\right], \quad B_{2}=\alpha \frac{1}{2} i\left[\Lambda_{2}, \Lambda_{3}\right]+\beta \frac{1}{2} i\left[\Lambda_{2}, \Lambda_{4}\right], \\
& B_{3}=-\alpha \frac{1}{2} i\left[\Lambda_{3}, \Lambda_{1}\right]+\beta \frac{1}{2} i\left[\Lambda_{3}, \Lambda_{2}\right], \quad B_{4}=\alpha \frac{1}{2} i\left[\Lambda_{4}, \Lambda_{1}\right]-\beta \frac{1}{2} i\left[\Lambda_{4}, \Lambda_{2}\right],
\end{aligned}
$$

where in turn $\alpha$ and $\beta$ are real positive parameters. The scaling transformations (2.15) changes $\alpha$ and $\beta$ to arbitrary positive values while a rotation (2.16) in 180 degrees does not change the sign of $B_{l}$. The particular signs of $\alpha$ and $\beta$ in (2.56) is directly related to our choice to let $x$ and $y$ represent positive metric states and $\lambda$ negative metric ones. By means of the particular solution

$$
|s\rangle=e^{B_{3}}|\phi\rangle_{3}, \quad B_{3}=-\alpha \frac{1}{2} i\left[\Lambda_{3}, \Lambda_{1}\right]+\alpha \frac{1}{2} i\left[\Lambda_{3}, \Lambda_{2}\right],
$$

we may now define transition amplitudes for imaginary times by $\left(\tau^{\prime}>\tau\right)$

$$
\left\langle s^{\prime}, \tau^{\prime} \mid s, \tau\right\rangle \equiv\left\langle s^{\prime}\left|e^{-H_{0}\left(\tau^{\prime}-\tau\right)}\right| s\right\rangle={ }_{3}\left\langle\phi^{\prime}\left|e^{-H_{0}\left(\tau^{\prime}-\tau\right)-2 \alpha \frac{1}{2} i\left[\Lambda_{3}, \Lambda_{1}\right]+2 \alpha \frac{1}{2} i\left[\Lambda_{3}, \Lambda_{2}\right]}\right| \phi\right\rangle_{3},
$$

where $H_{0}=H_{B R S T}^{\prime \prime}$ which commutes with $B_{l}$. Replacing $2 \alpha$ by $\tau^{\prime}-\tau$ we get the path integral expression (2.50) with imaginary times with the effective BRST invariant Hamiltonian

$$
H_{\mathrm{eff}}=\frac{1}{2}\left(p_{x}^{2}+p_{y}^{2}+P_{\lambda}^{2}\right)-i \mathcal{P}_{2} \mathcal{C}^{1}+i \overline{\mathcal{P}}^{1} \overline{\mathcal{C}}_{2}
$$

which is a natural geometric and gauge fixed Hamiltonian. In fact, the corresponding effective Lagrangian may be written

$$
L_{\mathrm{eff}}=\frac{1}{2}\left(\dot{x}^{2}+\dot{y}^{2}+\dot{\lambda}^{2}\right)+i \dot{\overline{\mathcal{C}}}_{a} \dot{\mathcal{C}}^{a}
$$

where we have made the following ghost redefinition: $\mathcal{C}^{1} \rightarrow \overline{\mathcal{P}}^{2}, \overline{\mathcal{C}}_{2} \rightarrow \mathcal{P}_{1}$. Notice that the conditions on $|\phi\rangle_{3}$ in (2.24) lead to the boundary conditions $\phi_{1}=0$ and $\lambda=0$ etc. in (2.50). 


\section{Application to Massive Vector Fields}

Encouraged by the previous results we treat now the massive vector field whose Lagrangian is given by (we use a space-like Minkowski metric)

$$
\mathcal{L}=-\frac{1}{4} F_{\mu \nu} F^{\mu \nu}-\frac{1}{2} m^{2} A_{\mu} A^{\mu}
$$

where $F_{\mu \nu}=\partial_{\mu} A_{\nu}-\partial_{\nu} A_{\mu}$. This Lagrangian leads to two second class constraints which eliminates $A^{0}$. However, these constraints do not have the necessary structure for a BRST quantization on an inner product space as described in the previous section. For that to be the case we need an additional unphysical degree of freedom. Since Lorentz covariance will require $A^{0}$ to be quantized with negative metric states we must add a bosonic unphysical scalar field, $\varphi$, which then must be quantized with positive metric states in order to be able to obtain inner product solutions. Since, we do not know off hand how this scalar should couple in the Lagrangian we choose for simplicity the most trivial coupling possible which leads to a constraint algebra like the one we had for the previous simple model. We consider therefore the following Lagrangian

$$
\mathcal{L}=-\frac{1}{4} F_{\mu \nu} F^{\mu \nu}-\frac{1}{2} m^{2} A_{\mu} A^{\mu}-\frac{1}{2} m^{2} \varphi^{2},
$$

where $\varphi$ is a real scalar field. The conjugate momenta to $A_{\mu}$ and $\varphi$ are given by

$$
\pi^{\mu}=\frac{\partial \mathcal{L}}{\partial \partial_{0} A_{\mu}}=F^{\mu 0}, \quad \pi=\frac{\partial \mathcal{L}}{\partial \partial_{0} \varphi}=0
$$

Hence, we have the primary constraints

$$
\phi_{1} \equiv \pi^{0}=0, \quad P_{\lambda} \equiv \pi=0 .
$$

The total Hamiltonian is

$$
H_{T}=\int \mathcal{H}_{T}(x) d^{3} x, \quad \mathcal{H}_{T}(x)=\mathcal{H}_{0}(x)+v(x) \pi^{0}(x)+w(x) \pi(x),
$$

where $\mathcal{H}_{0}(x)$ is the canonical energy density (up to a divergence)

$$
\mathcal{H}_{0}=\frac{1}{2} \pi_{i} \pi^{i}+A^{0} \partial_{i} \pi^{i}+\frac{1}{4} F_{i j} F^{i j}+\frac{1}{2} m^{2} A_{\mu} A^{\mu}+\frac{1}{2} m^{2} \varphi^{2} .
$$

The total Hamiltonian may be used to find out the consequences of the consistency conditions $\partial_{0} \pi^{0}=0$ and $\partial_{0} \pi=0$ for the primary constraints. By means of the Poisson bracket defined by

$$
\begin{aligned}
& \left.\left\{A^{\mu}(x), \pi_{\nu}(y)\right\}\right|_{x^{0}=y^{0}}=\delta_{\nu}^{\mu} \delta^{3}(x-y), \\
& \left.\{\varphi(x), \pi(y)\}\right|_{x^{0}=y^{0}}=\delta^{3}(x-y)
\end{aligned}
$$

we find

$$
\begin{aligned}
& \partial_{0} \pi^{0}=\left\{\pi^{0}, H_{T}\right\}=-\partial_{i} \pi^{i}+m^{2} A^{0} \\
& \partial_{0} \pi=\left\{\pi, H_{T}\right\}=-m^{2} \varphi
\end{aligned}
$$


Thus, consistency requires us to impose the secondary constraints

$$
\phi_{2} \equiv-\partial_{i} \pi^{i}+m^{2} A^{0}=0, \quad \lambda \equiv m^{2} \varphi=0 .
$$

The further consistency conditions $\partial_{0} \phi_{2}=0$ and $\partial_{0} \lambda=0$ require in turn that $v=\partial_{i} A^{i}$ and $w=0$ in the total Hamiltonian (3.5). The last term in (3.2) may obviously be removed without affecting the theory at the classical level. However, only with a term like this present do we have a constraint structure in exact correspondence to what we had for the simple model in the previous section and which is necessary for an inner product quantization. (The factor $m^{2}$ may be chosen arbitrary as will be shown in the next section.) The equal-time Poisson algebra of the constraints (3.4) and (3.9) are here

$$
\begin{aligned}
& \left\{\phi_{1}(x), \phi_{1}(y)\right\}=\left\{\phi_{2}(x), \phi_{2}(y)\right\}=\left\{P_{\lambda}(x), P_{\lambda}(y)\right\}=\{\lambda(x), \lambda(y)\}=0, \\
& \left\{\phi_{1}(x), \phi_{2}(y)\right\}=\left\{\lambda(x), P_{\lambda}(y)\right\}=m^{2} \delta^{3}(x-y) .
\end{aligned}
$$

We turn now to the quantum theory. From (3.7) we have the basic nonzero equal-time commutators

$$
\begin{aligned}
& {\left[A^{\mu}(x), \pi_{\nu}(y)\right]=i \delta_{\nu}^{\mu} \delta^{3}(x-y)} \\
& {[\varphi(x), \pi(y)]=i \delta^{3}(x-y),}
\end{aligned}
$$

which lead to

$$
\left[\phi_{1}(x), \phi_{2}(y)\right]=\left[\lambda(x), P_{\lambda}(y)\right]=i m^{2} \delta^{3}(x-y)
$$

for the constraint operators. In analogy with (2.8) we define the BRST operator to be

$$
\begin{aligned}
& Q=\int\left(\mathcal{C}^{a} \phi_{a}+\overline{\mathcal{P}}^{1} \lambda+\overline{\mathcal{P}}^{2} P_{\lambda}\right) d^{3} x \equiv \\
& \equiv \int\left\{\mathcal{C}^{1} \pi^{0}-\mathcal{C}^{2}\left(\partial_{i} \pi^{i}-m^{2} A^{0}\right)+\overline{\mathcal{P}}^{1} m^{2} \varphi+\overline{\mathcal{P}}^{2} \pi\right\} d^{3} x,
\end{aligned}
$$

where we have introduced the fermionic ghost field operators $\mathcal{C}^{a}, \mathcal{P}_{b}$ and $\overline{\mathcal{C}_{a}}, \overline{\mathcal{P}}^{b}$ satisfying

$$
\left[\mathcal{C}^{a}(x), \mathcal{P}_{b}(y)\right]=\delta_{b}^{a} \delta^{3}(x-y), \quad\left[\overline{\mathcal{C}}_{a}(x), \overline{\mathcal{P}}^{b}(y)\right]=\delta_{a}^{b} \delta^{3}(x-y) .
$$

We have then

$$
Q^{2}=i m^{2} \int\left(\mathcal{C}^{1} \mathcal{C}^{2}+\overline{\mathcal{P}}^{1} \overline{\mathcal{P}}^{2}\right) d^{3} x
$$

In analogy with the previous section we may e.g. define BRST invariant states by

$$
\begin{aligned}
& |s\rangle_{1,2}=\exp \left(\int\left(\alpha(x) \Lambda_{1}(x)+\beta(x) \Lambda_{2}(x)\right) d^{3} x\right)|\phi\rangle_{1,2} \\
& |s\rangle_{3,4}=\exp \left(\int\left(\alpha(x) \Lambda_{3}(x)+\beta(x) \Lambda_{4}(x)\right) d^{3} x\right)|\phi\rangle_{3,4},
\end{aligned}
$$

where $|\phi\rangle_{l}$ satisfy the local field conditions corresponding to (2.24). $\alpha(x)$ and $\beta(x)$ are parameter functions, and $\Lambda_{l}(x)$ are the following BRST invariant operator expressions

$$
\begin{aligned}
& \Lambda_{1}(x)=\phi_{2} \lambda-i m^{2} \overline{\mathcal{P}}^{2} \mathcal{P}_{2}+i m^{2} \mathcal{C}^{1} \overline{\mathcal{C}}_{1} \\
& \Lambda_{2}(x)=-\phi_{1} P_{\lambda}-i m^{2} \overline{\mathcal{P}}^{1} \mathcal{P}_{1}+i m^{2} \mathcal{C}^{2} \overline{\mathcal{C}}_{2}, \\
& \Lambda_{3}(x)=\phi_{2} P_{\lambda}+i m^{2} \overline{\mathcal{P}}^{1} \mathcal{P}_{2}+i m^{2} \mathcal{C}^{1} \overline{\mathcal{C}}_{2} \\
& \Lambda_{4}(x)=\phi_{1} \lambda-i m^{2} \mathcal{C}^{2} \overline{\mathcal{C}}_{1}-i m^{2} \overline{\mathcal{P}}^{2} \mathcal{P}_{1} .
\end{aligned}
$$


With appropriate choices of $|\phi\rangle_{l}$ the norms of $|s\rangle_{l}$ in (3.16) are finite. However, again the choices (3.16) are neither natural from a geometrical point of view nor does it lead to a regular effective theory.

A natural choice of a BRST invariant Hamiltonian is the following truncated version of the total Hamiltonian (3.5),

$$
\mathcal{H}^{\prime}(x)=\frac{1}{2} \pi_{i} \pi^{i}+\frac{1}{4} F_{i j} F^{i j}+\frac{1}{2} m^{2} A_{i} A^{i}-\partial_{i} \pi^{0} A^{i}+\frac{1}{2 m^{2}} \partial^{i} \pi^{0} \partial_{i} \pi^{0} .
$$

It commutes with all constraint operators and is therefore BRST invariant. If we then choose a BRST invariant solution of the form

$$
|s\rangle=e^{-\alpha B_{1}}|\phi\rangle_{1},
$$

where $\alpha$ is a positive constant and where

$$
\begin{aligned}
& B_{1} \equiv \frac{i}{2 m^{4}}\left[\int \Lambda_{1}(x) d^{3} x, \int\left(\Lambda_{3}(y)+\Lambda_{4}(y)\right) d^{3} y\right]= \\
& =\int\left\{-\frac{1}{2 m^{2}}\left(\partial_{i} \pi^{i}-m^{2} A^{0}\right)^{2}+\frac{1}{2} m^{2} \varphi^{2}-i \mathcal{C}^{1} \mathcal{P}_{2}-i \overline{\mathcal{P}}^{2} \overline{\mathcal{C}}_{1}\right\} d^{3} x
\end{aligned}
$$

we may define the transition amplitude for imaginary times by $\left(\tau^{\prime}>\tau\right)$

$$
\left\langle s^{\prime}, \tau^{\prime} \mid s, \tau\right\rangle \equiv\left\langle s^{\prime}\left|e^{-H^{\prime}\left(\tau^{\prime}-\tau\right)}\right| s\right\rangle={ }_{1}\left\langle\phi^{\prime}\left|e^{-H^{\prime}\left(\tau^{\prime}-\tau\right)-2 \alpha B_{1}}\right| \phi\right\rangle_{1} .
$$

Choosing $2 \alpha=\tau^{\prime}-\tau$ we may then extract an effective Hamiltonian in the path integral which contains the terms of the total Hamiltonian (3.5). However, it will also contain two additional terms. One term is $\partial_{i} \pi^{0} \partial^{i} \pi^{0} / 2 m^{2}$ which is a "gauge fixing" term that makes the Hamiltonian regular. The other term is $-\left(\partial_{i} \pi^{i}\right)^{2} / 2 m^{2}$ which is a genuine physical term which commutes with the BRST charge. The latter should not be in the effective Hamiltonian. Fortunately, this term is easily removed by replacing $\mathcal{H}^{\prime}$ in (3.18) by

$$
\mathcal{H}^{\prime \prime}=\mathcal{H}^{\prime}+\left(\partial_{i} \pi^{i}\right)^{2} / 2 m^{2},
$$

which also is BRST invariant and commutes with all constraints.

In a sense the construction given above is as successful as the corresponding construction for the simple model in the previous section. However, we notice that e.g. the term $\partial_{i} \pi^{0} \partial^{i} \pi^{0} / 2 m^{2}$ is not an appropriate term for an effective Hamiltonian since it does not lead to a "gauge fixing" term in the corresponding effective Lagrangian which is manifestly Lorentz invariant. In order to obtain such a Lagrangian we have to remove the term $\partial_{i} \pi^{0} \partial^{i} \pi^{0} / 2 m^{2}$ and insert new terms required by manifest Lorentz invariance. These terms should then only involve the constraint variables. The simplest choice is then to add the terms $-\left(\pi^{0}\right)^{2} / 2+\pi^{2} / 2+\partial_{i} \varphi \partial^{i} \varphi / 2$. This may be performed in a BRST invariant way by just replacing $B_{1}$ in 3.20 by $B_{1}^{\prime}$ given by

$$
\begin{aligned}
& B_{1}^{\prime}=B_{1}+\int\left\{-\frac{1}{2 m^{2}} \partial_{i} \pi^{0} \partial^{i} \pi^{0}-\frac{1}{2}\left(\pi^{0}\right)^{2}+\frac{1}{2} \pi^{2}+\frac{1}{2} \partial_{i} \varphi \partial^{i} \varphi+\right. \\
& \left.+i m^{2} \mathcal{C}^{2} \mathcal{P}_{1}+i m^{2} \overline{\mathcal{P}}^{1} \overline{\mathcal{C}}_{2}+i \partial_{i} \mathcal{C}^{2} \partial^{i} \mathcal{P}_{1}-\frac{i}{m^{2}} \partial_{i} \overline{\mathcal{P}}^{2} \partial^{i} \overline{\mathcal{C}}_{1}\right\} d^{3} x
\end{aligned}
$$

It is remarkable that the additional ghost terms which are required by BRST invariance also lead to a manifestly Lorentz invariant expression for the ghost terms in the effective Lagrangian. The final effective theory is described below. 


\section{Effective Theory for Massive Vector Fields}

Our manifestly Lorentz invariant effective Lagrangian for the massive vector field is given by

$$
\begin{aligned}
\mathcal{L}= & -\frac{1}{4} F_{\mu \nu} F^{\mu \nu}-\frac{1}{2} m^{2} A_{\mu} A^{\mu}-\frac{1}{2}\left(\partial_{\mu} A^{\mu}\right)^{2}-\frac{1}{2} \partial_{\mu} \varphi \partial^{\mu} \varphi-\frac{1}{2} \mu^{2} \varphi^{2}+ \\
& +i \partial_{\mu} \overline{\mathcal{C}}_{1} \partial^{\mu} \mathcal{C}^{1}+i m^{2} \overline{\mathcal{C}}_{1} \mathcal{C}^{1}+i \partial_{\mu} \overline{\mathcal{C}}_{2} \partial^{\mu} \mathcal{C}^{2}+i \mu^{2} \overline{\mathcal{C}}_{2} \mathcal{C}^{2}
\end{aligned}
$$

where we have redefined the ghost fields as compared to the previous section (see below). This Lagrangian yields the simple equations

$$
\begin{aligned}
& \left(\square-m^{2}\right) A^{\mu}=\left(\square-m^{2}\right) \mathcal{C}^{1}=\left(\square-m^{2}\right) \overline{\mathcal{C}}_{1}=0, \\
& \left(\square-\mu^{2}\right) \varphi=\left(\square-\mu^{2}\right) \mathcal{C}^{2}=\left(\square-\mu^{2}\right) \overline{\mathcal{C}}_{2}=0 .
\end{aligned}
$$

Thus, the five bosonic and four fermionic degrees of freedom all satisfy a massive free equation. The Lagrangian (4.1) is quasi-invariant under the generalized BRST transformation

$$
\begin{aligned}
& s A^{\mu}=-\partial^{\mu} \mathcal{C}^{1}, \quad s \overline{\mathcal{C}}_{1}=-i \partial_{\mu} A^{\mu}, \\
& s \varphi=-m \mathcal{C}^{2}, \quad s \overline{\mathcal{C}}_{2}=-i m \varphi, \quad s \mathcal{C}^{a}=0 .
\end{aligned}
$$

which only is nilpotent on the matter fields $A^{\mu}$ and $\varphi$. In fact, we have

$$
s^{2} A^{\mu}=s^{2} \varphi=0, \quad s^{2} \overline{\mathcal{C}}_{1}=i \square \mathcal{C}^{1} \approx i m^{2} \mathcal{C}^{1}, \quad s^{2} \overline{\mathcal{C}}_{2}=i m^{2} \mathcal{C}^{2} .
$$

The conserved current density corresponding to the above BRST invariance is

$$
J^{\mu}=F^{\mu \nu} \partial_{\nu} \mathcal{C}^{1}-m^{2} A^{\mu} \mathcal{C}^{1}+\left(\partial_{\nu} A^{\nu}\right) \partial^{\mu} \mathcal{C}^{1}+m \varphi \stackrel{\leftrightarrow}{\partial^{\mu}} \mathcal{C}^{2}
$$

In order to demonstrate the equivalence with what we had in the previous section we perform a transition to the corresponding Hamiltonian formulation. We define then the conjugate momenta to the field variable in (4.1) by

$$
\begin{aligned}
& \pi^{\mu}=\frac{\partial \mathcal{L}}{\partial \partial_{0} A_{\mu}}=F^{\mu 0}+\partial_{\nu} A^{\nu} \eta^{\mu 0}, \quad \pi=\frac{\partial \mathcal{L}}{\partial \partial_{0} \varphi}=\partial^{0} \varphi \\
& \mathcal{P}_{a}=i \frac{\partial \mathcal{L}}{\partial \partial_{0} \mathcal{C}^{a}}=-\partial_{0} \overline{\mathcal{C}}_{a}, \quad \overline{\mathcal{P}}^{a}=i \frac{\partial \mathcal{L}}{\partial \partial_{0} \overline{\mathcal{C}}_{a}}=\partial_{0} \mathcal{C}^{a}
\end{aligned}
$$

where $\eta^{\mu \nu}$ is the space-like Minkowski metric. The Hamiltonian density is given by

$$
\begin{aligned}
\mathcal{H}= & \pi^{\mu} \partial_{0} A_{\mu}+\pi \partial_{0} \varphi+i \mathcal{P}_{a} \partial_{0} \mathcal{C}^{a}+i \overline{\mathcal{P}}^{a} \partial_{0} \overline{\mathcal{C}}_{a}-\mathcal{L}=\frac{1}{2} \pi^{i} \pi_{i}-\frac{1}{2}\left(\pi^{0}\right)^{2}+ \\
& +\frac{1}{4} F^{i j} F_{i j}+\frac{1}{2} m^{2} A^{\mu} A_{\mu}-\pi^{i} \partial_{i} A^{0}+\pi^{0} \partial_{i} A^{i}+\frac{1}{2} \pi^{2}+\frac{1}{2} \mu^{2} \varphi^{2}+ \\
& +\frac{1}{2} \partial_{i} \varphi \partial^{i} \varphi+i \mathcal{P}_{a} \overline{\mathcal{P}}^{a}-i \partial_{i} \overline{\mathcal{C}}_{a} \partial^{i} \mathcal{C}^{a}-i m^{2} \overline{\mathcal{C}}_{1} \mathcal{C}^{1}-i \mu^{2} \overline{\mathcal{C}}_{2} \mathcal{C}^{2}
\end{aligned}
$$

From (4.5) we find the BRST charge

$$
Q=\int J^{0}(x) d^{3} x=\int\left\{\left(\partial_{i} \pi^{i}-m^{2} A^{0}\right) \mathcal{C}^{1}-\pi^{0} \overline{\mathcal{P}}^{1}+m \varphi \overline{\mathcal{P}}^{2}-m \pi \mathcal{C}^{2}\right\} d^{3} x,
$$


which generates the BRST transformation (4.3) in terms of the Poisson bracket defined by $(3.7)$ and

$$
\begin{aligned}
& \left.\left\{\mathcal{C}^{a}(x), \mathcal{P}_{b}(y)\right\}\right|_{x^{0}=y^{0}}=-i \delta_{b}^{a} \delta^{3}(x-y), \\
& \left.\left\{\overline{\mathcal{C}}_{a}(x), \overline{\mathcal{P}}^{b}(y)\right\}\right|_{x^{0}=y^{0}}=-i \delta_{a}^{b} \delta^{3}(x-y) .
\end{aligned}
$$

Comparison with (3.13) tells us that the ghost fields $\left\{\mathcal{C}^{1}, \mathcal{C}^{2}, \overline{\mathcal{C}}_{1}, \overline{\mathcal{C}}_{2}\right\}$ here are equal to the ghost fields $\left\{-\mathcal{C}^{2},-\frac{1}{m} \overline{\mathcal{P}}^{2},-\mathcal{P}_{1}, \frac{1}{m} \overline{\mathcal{C}}_{1}\right\}$ in the previous section. Notice that the only BRST invariant canonical conjugate variables are

$$
\tilde{A}^{i} \equiv A^{i}-\frac{1}{m^{2}} \partial^{i} \pi^{0}, \quad \tilde{\pi}^{i} \equiv \pi^{i}
$$

which exactly represent the degrees of freedom of a massive vector field. In the quantum theory the degrees of freedom are governed by BRST triplets in involution. As was shown in section 2 we have here two sets of commuting BRST triplets. They are

$$
\begin{aligned}
& \text { 1) }\left\{\overline{\mathcal{C}}_{1}, \pi^{0}, m^{2} \mathcal{C}^{1}\right\} \text { and }\left\{\mathcal{P}_{2}, m \pi, m^{2} \overline{\mathcal{P}}^{2}\right\} \\
& \text { 2) }\left\{\mathcal{P}_{1}, \partial_{i} \pi^{i}-m^{2} A^{0}, m^{2} \overline{\mathcal{P}}^{1}\right\} \text { and }\left\{\overline{\mathcal{C}}_{2}, m \varphi, m^{2} \mathcal{C}^{2}\right\}
\end{aligned}
$$

or combinations of these two sets. They remove the $\varphi$ and $A^{0}$-fields and all the ghost variables leaving only the degrees of freedom of a massive vector field.

It is interesting to see what happens in the massless limit. In the limit $m \rightarrow 0$ the Lagrangian (4.1) reduces to

$$
\begin{aligned}
& \mathcal{L}_{0}=-\frac{1}{4} F_{\mu \nu} F^{\mu \nu}-\frac{1}{2}\left(\partial_{\mu} A^{\mu}\right)^{2}+i \partial_{\mu} \overline{\mathcal{C}}_{1} \partial^{\mu} \mathcal{C}^{1}+i \partial_{\mu} \overline{\mathcal{C}}_{2} \partial^{\mu} \mathcal{C}^{2}- \\
& -\frac{1}{2} \partial_{\mu} \varphi \partial^{\mu} \varphi-\frac{1}{2} \mu^{2} \varphi^{2}+i \mu^{2} \overline{\mathcal{C}}_{2} \mathcal{C}^{2}
\end{aligned}
$$

which is invariant under the nilpotent BRST transformations

$$
\begin{aligned}
& s A^{\mu}=-\partial^{\mu} \mathcal{C}^{1}, \quad s \overline{\mathcal{C}}_{1}=-i \partial_{\mu} A^{\mu}, \\
& s \varphi=s \overline{\mathcal{C}}_{2}=s \mathcal{C}^{a}=0 .
\end{aligned}
$$

The ghosts $\mathcal{C}^{1}$ and $\overline{\mathcal{C}}_{1}$ are obviously reduced to the standard ghost, antighost fields in QED while $\varphi, \mathcal{C}^{2}$ and $\overline{\mathcal{C}}_{2}$ are turned into decoupled BRST invariant field variables with mass $\mu$ (BRST singlets). The BRST current (4.5) and the BRST charge (4.8) reduce to the one for a massless vector field. Now the limit seems to lead to an inconsistent theory unless we remove the ghosts $\mathcal{C}^{2}$ and $\overline{\mathcal{C}}_{2}$. However, in the quantum theory this is automatic since the two sets of commuting BRST triplets reduce to the BRST doublets of the massless theory and to BRST singlets which remove the ghosts $\mathcal{C}^{2}$ and $\overline{\mathcal{C}}_{2}$. Thus, the massless limit is consistent and has exactly as many degrees of freedom as the massive case since the scalar field $\varphi$ then becomes physical.

The above model is quite similar to the Stückelberg model for a free massive vector field. The latter is described by the Lagrangian

$$
\mathcal{L}_{1}=-\frac{1}{4} F_{\mu \nu} F^{\mu \nu}-\frac{1}{2} m^{2}\left(A_{\mu}-\frac{1}{m} \partial_{\mu} \varphi\right)\left(A^{\mu}-\frac{1}{m} \partial^{\mu} \varphi\right)
$$


which obviously is invariant under the gauge transformation $A^{\mu} \rightarrow A^{\mu}+\partial^{\mu} \Lambda, \varphi \rightarrow \varphi+m \Lambda$. This is an example of a conversion of second class constraints to first class ones. In fact, it is a particular example of the general conversion mechanism given in 2]. To see this one may consider the equivalent Lagrangian

$$
\mathcal{L}_{2}=-\frac{1}{4} F_{\mu \nu} F^{\mu \nu}-\frac{1}{2} m^{2} A_{\mu} A^{\mu}-\frac{1}{2} \partial^{\mu} \varphi \partial_{\mu} \varphi-m \partial_{\mu} A^{\mu} \varphi
$$

It yields the constraints

$$
\pi^{0}+m \varphi=0, \quad \partial_{i} \pi^{i}-m^{2} A^{0}-m \pi=0,
$$

which are the original constraints for the massive vector field obtained from (3.1) modified with linear terms in the canonical conjugate variables of the scalar field in such a way that the resulting constraints become first class ones. The corresponding effective theory is described by the Lagrangian

$$
\begin{aligned}
\mathcal{L}_{S}= & -\frac{1}{4} F_{\mu \nu} F^{\mu \nu}-\frac{1}{2} m^{2} A_{\mu} A^{\mu}-\frac{1}{2}\left(\partial_{\mu} A^{\mu}\right)^{2}- \\
& -\frac{1}{2} \partial_{\mu} \varphi \partial^{\mu} \varphi-\frac{1}{2} m^{2} \varphi^{2}+i \partial_{\mu} \overline{\mathcal{C}} \partial^{\mu} \mathcal{C}+i m^{2} \overline{\mathcal{C}} \mathcal{C},
\end{aligned}
$$

which is invariant under the nilpotent BRST transformation

$$
\begin{aligned}
& s A^{\mu}=-\partial^{\mu} \mathcal{C}, \quad s \overline{\mathcal{C}}=-i\left(\partial_{\mu} A^{\mu}-m \varphi\right), \\
& s \varphi=-m \mathcal{C}, \quad s \mathcal{C}=0 .
\end{aligned}
$$

The corresponding conserved BRST current is

$$
J^{\mu}=F^{\mu \nu} \partial_{\nu} \mathcal{C}-m^{2} A^{\mu} \mathcal{C}+\left(\partial_{\nu} A^{\nu}\right) \partial^{\mu} \mathcal{C}+m \varphi \stackrel{\leftrightarrow}{\partial^{\mu}} \mathcal{C}
$$

This is obviously very similar properties to what we had before. The differences are that the number of ghost fields in (4.1) are twice as many as in (4.17), the masses of the scalar and vector fields are the same in (4.17) while they may be different in (4.1). The Stückelberg model is obviously preferable at the free level due to its exact BRST properties. However, both models are consistent.

\section{Conclusions}

In the present paper we have developed the generalized BRST method for second class constraints which was proposed in [9, 10]. This we have done by a careful analysis of a simple model with finite number of degrees of freedom. In this process we have discovered what we think are rather general features of this approach. We have e.g. demonstrated that the method works provided the number of unphysical bosonic degrees of freedom is even. (Half of the degrees of freedom must be quantized with indefinite metric states and half with positive metric states.) The physical states have the form

$$
|p h y s\rangle=e^{\Lambda}|\phi\rangle,
$$

where $\Lambda$ is BRST invariant and the properties of $|\phi\rangle$ is determined by hermitian BRST triplets $(|\phi\rangle$ is then BRST invariant). Also in ordinary BRST quantization the physical 
states have the form (5.1) but then $|\phi\rangle$ is governed by hermitian BRST doublets and $\Lambda$ is given by $[\psi, Q]$ where $\psi$ is a hermitian gauge fixing fermion. In the second class case there is no gauge freedom in $\Lambda$. However, there are different forms of $\Lambda$ which yields different effective theories. The precise form of this freedom we have not pinned down but it is quite limited. The possible forms of $\Lambda$ are connected to the precise choice of $|\phi\rangle$. In the ordinary case $|\phi\rangle$ may be defined to be an inner product state due to the supersymmetry of the unphysical degrees of freedom. This is not the case here since the number of ghosts is doubled. In the considered bosonic models $|\phi\rangle$ is a zero norm state. The extra ghosts we have in the effective actions provide for the necessary measure factor. The only case in which $|\phi\rangle$ could be defined as an inner product state is when we have a supersymmetric set of second class constraints. Our derivations of effective Hamiltonians were rather awkward and cumbersome. One reason is that we had to use a method which is not fully developed even in the ordinary case. It is clear that this method may be considerably improved. We need simple algorithms. Therefore, from a mathematical point of view there are much to be done. However, we do not expect that the treatment of second class constraints can ever be as beautiful as the one for first class constraints, i.e. general gauge theories.

When we applied the formalism as developed in section 2 to a free massive vector field we found a simple effective Lagrangian which was very similar to the one from the Stückelberg model. Notice, however, that the additional scalar field was in our case introduced in order to have inner product states while in the Stückelberg model it is introduced in order to restore gauge invariance. Although the Stückelberg formalism is preferable to our formulation in the free case, this might not be so in the interaction case. Notice that both formulations are consistent. Since the basic motivation behind our work was to find new ways to quantize massive Yang-Mills in a consistent fashion we have at least made one step forward. One may notice that the generalization of the Stückelberg model to the massive Yang-Mills leads to nonpolynomial terms in the scalars which are nonrenormalizable [5]. Since our model is not based on the Stückelberg transformation it is not unlikely that one may construct a massive Yang-Mills with polynomial interaction terms which are renormalizable. Maybe the old Curci-Ferrari model treated in [11] is such a theory even when additional scalars are added.

\section{Acknowledgements}

R.M. would like to thank Igor Batalin and Simon Lyakhovich for stimulating discussions on second class constraints. He would also like to thank the Invitation Fellowship Program of the Japanese Society for the Promotion of Science (JSPS) for his stay in Japan.

\section{References}

[1] I. A. Batalin and G. A Vilkovisky, Phys. Lett. B69, 309 (1977)

E. S. Fradkin T. E. Fradkina, Phys. Lett. B72, 343 (1978)

I. A. Batalin and E. S. Fradkin, Phys. Lett. B122, 157 (1983) 
[2] I. A. Batalin and E. S. Fradkin, Nucl. Phys. B279, 514 (1987)

[3] E. C. G. Stückelberg, Helv. Phys. Acta 11, 299 (1933)

[4] T. Kunimasa and T. Goto, Prog. Theor. Phys. 37, 452 (1967)

[5] T. Appelquist and C. Bernard, Phys. Rev. D22, 200 (1980)

A. Burnel, Phys. Rev. D33, 2985 (1986)

N. Dragon, T. Hurth and P. v. Nieuwenhuizen, Polynomial Form of the Stueckelberg Model Preprint hep-th/9703017

[6] P. Senjanovic, Ann. Phys. 100, 227 (1976)

[7] I. A. Batalin, I. V. Tyutin and S. L. Lyakhovich, Mod. Phys. Lett. A 7, 1931 (1992)

[8] I. A. Batalin, I. V. Tyutin and S. L. Lyakhovich, Int. J. Mod. Phys. A 10, 1917 (1995)

[9] R. Marnelius, Nucl. Phys. B294, 685 (1987)

R. Marnelius, Generalized BRST quantization., Proc. Int. meeting on geometrical and algebraic aspects of nonlinear field theory. Amalfi, Italy, 1988, Ed S. De Filippo, M. Marinaro, G. Marmo and G. Vilasi North-Holland, Amsterdam, 1989.

[10] R. Marnelius, Nucl. Phys. B370, 165 (1992)

[11] I. Ojima, Z. Phys. C13, 173 (1982)

[12] R. Marnelius, Nucl. Phys. B395, 647 (1993)

[13] R. Marnelius, Phys. Lett. B318, 92 (1993)

[14] R. Marnelius, Nucl. Phys. B418, 353 (1994)

[15] I. Batalin and R. Marnelius, Nucl. Phys. B442, 669 (1995)

[16] G. Fülöp and R. Marnelius, Nucl. Phys. B456, 442 (1995)

[17] R. Marnelius, Time evolution in general gauge theories on inner product spaces, Nucl. Phys. B (in press)

[18] R. Marnelius, Phys. Lett. B172, 337 (1986)

R. Marnelius, Relativistic strings in subcritical dimensions - the power of BRS quantization, Published in Constraint's theory and relativistic dynamics. Eds. G. Longhi and L. Lusanna, World Scientific (1987)

[19] R. Marnelius and Sh. M. Shvartsman, Nucl. Phys. B430, 153 (1994) 